\title{
An Integrative Review of Experience of Well-Being and Happiness: Insights from Older Adults in the Community
}

\author{
Jurawan Jitdorn", Chomchuen Somprasert, \\ Puangpaka Kongvattananon and Rangsiman Soonthornchaiya
}

Faculty of Nursing, Thammasat University, Pathum Thani 12121, Thailand

('Corresponding author's e-mail: jurawan027@nurse.tu.ac.th)

Received: 22 May 2020, Revised: 23 May 2021, Accepted: 24 May 2021

\begin{abstract}
Well-being and happiness are the ultimate goals of older people. Having a good quality of life is very important for this age group, in which well-being can be predicted through life experiences. Research on the older adult group demonstrates that well-being is positively associated with happiness. In turn, happiness is linked with better coping, lower morbidity and lower mortality. Therefore, studying to understand this phenomenon, according to the experiences and perceptions of older adults, is the key to preparing for an aging society. The objective of this integrative review for nurses was to synthesize information from academic literature to provide insight into the experience and perception of well-being and happiness in older adults. The authors searched 5 databases - CINAHL, PUBMED, CLINICAL KEY, SCOPUS and SAGE journals - using the following key search terms: "(experience or perception or perspectives), AND (happiness or well-being), AND (older adults or elderly or late adulthood)." Only articles published between January 2010 and April 2020 were considered. The preferred reporting items for the Joanna Briggs Institute Critical Appraisal tools were adopted for quality assessment. The authors finally selected 12 out of 651 references for integrative review.

The results show that the key points of view associated with the well-being and happiness of older people consist of 3 important issues. The first issue includes the challenges and concerns about late life involving independence, life quality, fear of squandering free time, being left out, and having a good death. Second are the strategies for living a meaningful late life through doing regular daily activities, creating selfesteem, finding life satisfaction, engaging in subjectively perceived activities, being optimistic, relying on religious attachment, and setting life goals. The last issue includes resources and support for older adults to reach their goals, which are family support, social support, and religious support. A challenge for future research is how to create interventions that promote the independence that leads to freedom in late life.
\end{abstract}

Keywords: Experience, Happiness, Integrative review, Older adults in the community, Well-Being.

\section{Introduction}

The World Health Organization (WHO) reports that people worldwide are living longer, resulting in the growth of the older population. Between 2015 and 2050, the proportion of the world's population over 60 years old will nearly double, from 12.0 to $22.0 \%$. Naturally, older adults are declining in their physical and cognitive efficiency. There are risk groups for health problems, most of which are chronic diseases caused by the degeneration of various organs. This has resulted in a continuous increase in dependency rates, from $10.70 \%$ in 1994 to $22.30 \%$ in 2014 [1]. Considering the increase in life expectancy, the researchers are interested in studying quality of life in older adults, with focus on maintaining and promoting well-being and happiness in the old age group. For the purposes of this discussion, an "older" adult is defined as someone age 60 and older [1,2].

Well-being is an experience of good health, happiness, and prosperity that seems to be everyone's dream. It includes good mental health, high life satisfaction, full life meaning or purpose, good social relationships, and the ability to manage stress. Unfortunately, well-being seems to be generally declining, and can be difficult to improve without certain skills, particularly among the older adult group, where there is increasing opportunity for negative experiences that could threaten physical and mental wellbeing. Aging can be associated with losses and changes in some life domains [3]. Well-being in this age group is particularly important and can be judged based on happiness and life satisfaction. Among older 
people, happiness is linked with coping ability, which will help lower illness and mortality rates [4]. The literature review found that the study of happiness and well-being meant that people assessed their lives based largely on 3 main structures: Life Satisfaction - understood as the cognitive appraisal of life itself and positive and negative affect, which indicate the immediate response to the good and bad features of life [5].

Although many studies have been conducted in this area, there is still a gap in studies focusing on in-depth issues from a variety of experiences and different perspectives of older adult groups with diverse socio-cultural demographics. Most of the existing literature has disregarded the topic of continuous development in old age. However, it is possible and certain that the evolution of perceptions and thought continue throughout one's lifespan. Therefore, it is important to understand well-being in older adults, aged $\geq 60$ years, based on their experiences, perspectives, and daily activities, which may help in observing and understanding changes in the levels of life happiness in these older adults. Hence, an integrative review of the literature is conducted to synthesize what is currently known on this issue. The integrative review will establish current knowledge on this specific topic, because it will clarify the identification, analysis, and synthesis of independent study on the same subject, thus contributing to reflecting the possible benefits in the quality of care delivered to patients [6].

\section{Methods}

The method used in this study was an integrative review. This methodology is the broadest type of research review method and allows concurrent inclusion of quantitative and qualitative research to allow fuller understanding of a phenomenon of concern (well-being and happiness in older adults). Whittemore and Knafl [7] advised an integrative approach design, consisting of 5 stages, including problem detection, literature search, data assessment, data analysis (results), and presentation (conclusion), to improve process consistency while performing an integrative review.

\section{Problem identification}

While aging is a natural process in our lives, older adults should be involved in creating their wellbeing and happiness in life. Therefore, conversations about the well-being and happiness of older adults converging with the search for experiences, perceptions, and perspectives will help us understand more deeply and lead to the discovery of ways to improve the future. It is important to start with a welldeveloped question, such as, "What is the experience and perception of well-being and happiness in older adults?", which was developed with the modified PICO framework [8]. The PICO framework has details as follows: Population (P) is older adults; phenomenon of interest (I) means experiences, perceptions, perspectives; context $(\mathrm{CO})$ is experiences with well-being and happiness among the older adults in the community.

\section{Literature search}

The second stage of the integrative review is a literature search. The terms used during the search process included experience or perception or perspectives and happiness or well-being and older adults or elderly or late adulthood. Search limits included publications from 2010 through 2020, using the CINAHL, PUBMED, CLINICAL KEY, SCOPUS, SAGE journal databases. Relevant bibliographies were also scanned, and relevant references were added. The internet was searched for grey literature.

Study selection was undertaken in 2 phases. First, each researcher screened the relevance of the titles and abstracts. The inclusion criteria of the survey studies were as follows: (a) samples were older people, aged $\geq 60$ years, (b) the research objective was to synthesize knowledge about experience and perception on happiness and well-being in older adults from their perspectives, (c) the study data were from qualitative and quantitative research in cross-sectional design studies and mixed methods studies, and (d) the abstracts were presented in Thai and English. Non-referenced articles and dissertations were excluded from the review. Second, when the documents appeared to meet the inclusion criteria, the 2 researchers acquired the full text and screened them together. Any conflicts were solved by obtaining consensus from the third researcher.

\section{Data evaluation}

The quality of the studies was evaluated by using the Joanna Briggs Institute Critical Appraisal (JBI) tools. The tools of the JBI appraisal checklist for qualitative research contain 10 methodological items for qualitative studies, while the tools of the JBI appraisal checklist for analytical cross-sectional studies and mixed methods studies contain 8 methodological items for quantitative studies. The writers in 
this process used the critical evaluation methods specific to each article's methodology. Next, all articles were imported into the analysis process [9].

\section{Data analysis and interpretation}

Data analysis followed a pre-determined 4-step cycle of data display, data grouping, data categorization and data synthesis, according to integrative review methodology. This step-by-step approach made it easier to identify patterns of change and relationships from the extracted data. The validation occurred from a systematic examination of the main literature that was both internal and crossgroup [7]. The interpretation of data was from a discussion of the authors before separating the data into the attributes of the researching phenomenon, focusing on the purpose of the article, extracting according to the study objectives, finding the study results, summarizing the data from each study, and comparing the main results using the analysis process. Afterward, important components were combined and conclusions were drawn from each subgroup to be incorporated into this study topic.

\section{Results and discussion}

\section{Results}

An initial search of the aforementioned databases yielded 646 articles. Further searching of the identified records through other sources of the included studies found another 5 related articles to be included in the screening process. Three articles were not included due to duplications. The inclusion and exclusion criteria were applied to 648 articles. After screening, 623 articles were excluded, because they were not related to the experience or perception of well-being and happiness in late adulthood. After further review of 25 articles, 13 articles were separated, because the content did not match the educational protocol; the results of the study did not answer the study questions; the samples were less than 60 years old, and the articles were not published in English or Thai. The remaining 12 articles entered the final round. The process of data extraction following the PRISMA flow chart [10,11] is illustrated in Figure 1

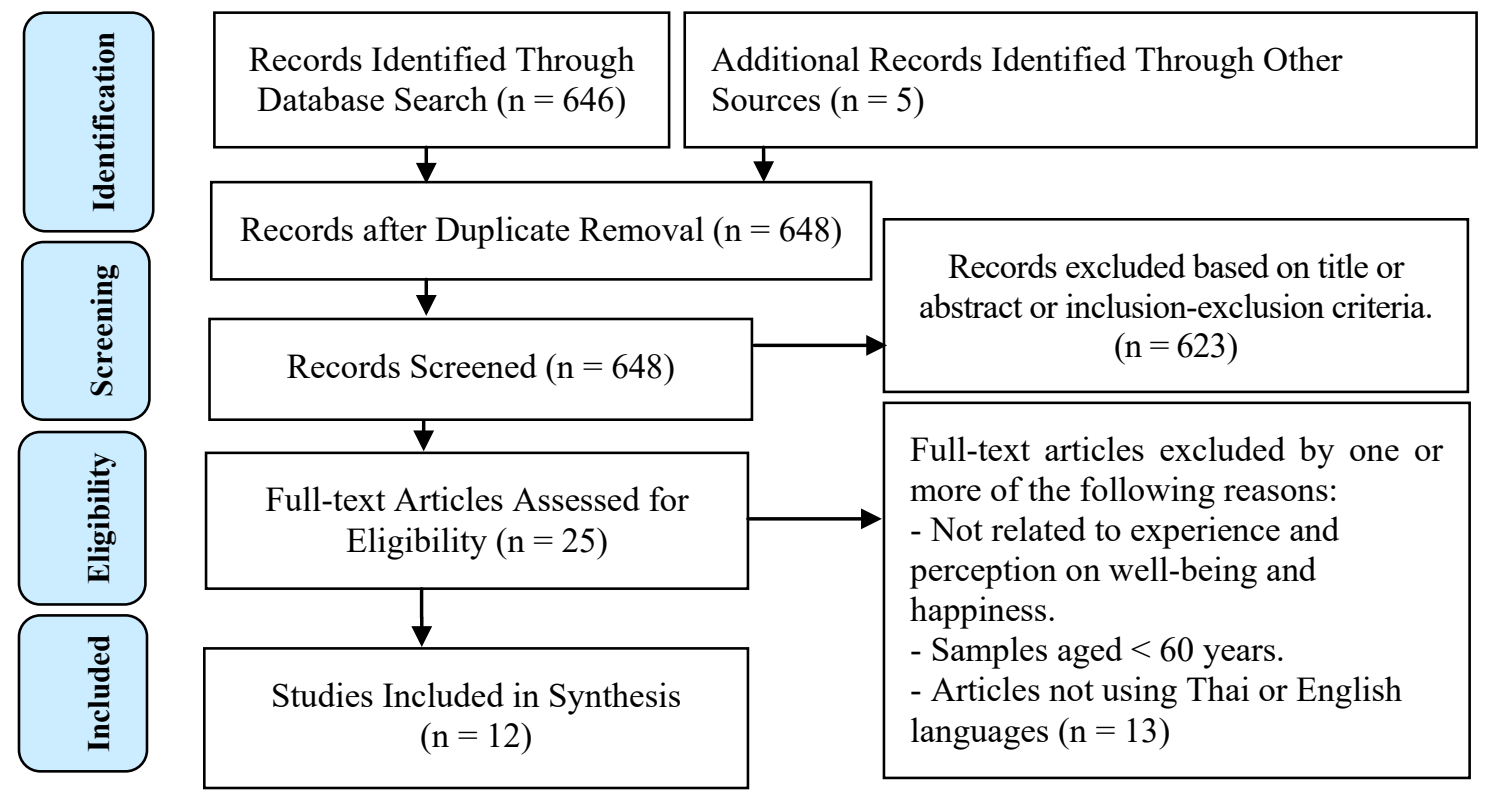

Figure 1 Flow diagram showing search strategies and results.

The present review included 6 published quantitative studies (cross-sectional design studies) from 2010 - 2018. The sample sizes of the studies varied from 70 to 20,351 participants. Most participants lived in their houses in the community. Six qualitative studies were published from 2012 - 2019. The sample sizes of the studies varied from 12 to 84 participants. Most participants lived in the community. One study was conducted with participants living in a nursing home; the participants were $60-100+$ years of age. The integrative review of content from quantitative and qualitative research could be summarized through the 
meaning of well-being and happiness in older adults, and there were 3 key points of view associated with wellbeing and happiness: Insights from older adults, as follow:

\section{Meaning of well-being in older adults}

The well-being of older adults related to physical, psychological, emotional, social and spiritual dimensions, which meant understanding life purpose, ability to communicate with others, ability to improve self-care and functioning, being able to maintain personal dignity, and contribution to society. However, the most important aspect for older adults' well-being is the ability to maintain maximum independence [12-15].

\section{Meaning of happiness in older adults}

From the perspectives of older adults, life happiness is related to good memories of childhood and adulthood, good feelings, lack of anger, lack of aggression, good health, good moments in everyday life, positive relationships with family and friends, life satisfaction, self-acceptance and self-esteem [12,14,15].

\section{Key points of view associated with well-being and happiness: Insights into older adults involve 3 main viewpoints \\ Life challenges and concerns of older adults}

From the data synthesis, the literature of 7 articles revealed that, when asked about well-being and life happiness from the experiences of older adults, the older adults mentioned their current stage of life, life needs, personal independence, being happy and having good life, fear of squandering free time or being missed out and having ideas about a good death in greater detail, as follows:

\section{Needing independence concerning various aspects of life}

The loss of control also reflected the reduction of personal independence, especially in the participants' physical condition, in terms of fear of deterioration and physical pain and suffering, which were expressed as limiting the ability of participants [16]. Loss of physical function in the process of aging, such as loss of sight and limited mobility, affect independence in self-management in the late elderly [12]. Nosraty et al. [13] found that older adults were considered to need physical independence, financial independence, autonomy, decision-making, and self-mastery, which affected their well-being of life. From the reviews, it was found that reports of daily independent goal progress could predict improvement of the mental and physical well-being of older adults on the same day and the next day.

\section{Having ideas about a good death}

As age increases, older adults need a decrease in their fear of death [17]. The findings of Nosraty et al. [13] showed that maintaining dignity and independence was part of the quality of both life and death. This was interpreted from an interview with an elderly man living with a spouse without help from home. "What can I say about it, not much ... I'm in balance mentally, not afraid of death."

\section{Being happy and having a good life}

There were stories about having a good and happy life in many aspects based on the lives of the older adults, which focused on the things the older adults liked, and good experiences in past life, such as childhood, working outside, marriage, good health, and having a happy family. On the other hand, the older adults in the study also reported receiving too little time from family as a source of disappointment [12]. Rinnan [14] stated that positive relations, a sense of belonging, sources of meaning, moments of feeling well, and acceptance constituted the conceptualization of the Joy of Life (JOL) phenomenon.

Use of worthwhile time and fear of squandering free time and being missed out

Older adults were afraid of missing out on the desire to do as much as possible, to learn as much as they could, and to gain as many new experiences as their time allowed on this earth. Therefore, they would like to use the rest of their time to increase the benefits of others, according to their ability, by participating things such as volunteering for charity work, group activities, and other activities to fill up a day with happiness and meaningful goals in life [16].

\section{Strategies for a meaningful life in older adults}

A strategy is an action taken to achieve a goal and could be defined as "directions and components to achieve a desired state in the future" [18]. A literature review of 10 articles revealed that content about the experiences and perspectives of older adults related to strategies that helped make meaning of life at this 
age, and included daily routines, creating self-esteem and life satisfaction, engagement, subjectively perceived activities, optimism, attachment to God, and having a goal in life, which are described as follows:

\section{Doing regular daily activities}

Participation in daily activities, listening to the radio, reading newspapers, meeting other seniors, and not having to rely on family and nurses are sources of meaning in life and happiness [14]. In addition, doing daily activities alone also helped to promote subjective memory, which was related to the subjective well-being of the older adults in this study. Determining daily life activities helped them sense their goals and objectives in waking up in the morning to do something. In addition, keeping an active lifestyle in older adults helped to foster development and growth, despite the difficulties and struggles faced in life. An active lifestyle was a buffer for boredom, an antidote for withdrawal from life, and reduced depression among the older adults [16].

\section{Creating self-esteem and life satisfaction}

The older adults in this study expressed the importance of being something of value to others in some way, stating that caring for or helping others gave them a sense of acceptance and value which created self-esteem [14]. According to the findings of a study by Roman et al. [19], "Life satisfaction was associated with increased age; male gender; participation in social organizations; being married or living as a couple; higher education level; higher income; good perceived health status, and better health care." The subjective meaning: well-being was improved by variables in the health aspect, perception of high health conditions, private health insurance, and average weight gain.

\section{Engagement and subjectively perceived social activities}

From the literature review, it was found that the achievement of world peace was a primary value in older adults. In terms of the activities of older adults aged 100+ years, a 100-year-old woman stated, "I still don't think of myself as too old to do anything" [12]. This was consistent with the study of Kavcic and Avsec [15], who said, "The results highlight the importance of engagement and subjectively perceived activities throughout 1 day, which were investigated and found to be associated with all aspects of emotional, psychological and social well-being in late adulthood." The study of Gonzalez-Herero and Garcia-Martin [5] confirmed the importance of participation in social activities to increase personal wellbeing in middle and old age. The participation in social activities among older people had relationships with increases in mass communication use, life satisfaction, high positive impact, and low negative impact.

\section{Learning to be optimistic}

Establishing strategies for accepting situations arising in life and adopting a good lifestyle to deal with loss, loneliness, and vulnerability are key strategies for older adults. The older adults in this study had awareness and created positive viewpoint, even if they had weak health and knew they were near the end of their lives [14]. Over time, as age increased, the greater experience of the late seniors resulted in feelings of acceptance of others and their life situations, especially feelings of being less judgmental and more open-minded in listening to others [17]. In this case, optimism included various dimensions of forgiveness that had a positive relationship with psychological well-being, life satisfaction, and selfesteem, and negative relationships with depression in older adults. Optimism is a powerful predictor of well-being in older adults, and the main predictors of well-being in this study were both perceived social support and perception of control. Friends and family were sources that helped promote optimism by increasing the awareness of the older adults. This made them happy with a sense of purpose and meaning. Thus, optimism was the result of creating a feeling that they had control of their environments [20].

\section{Obtaining an attachment to God for psychological well-being in older adults}

It was found that older people who had an attachment to God felt more secure (not worried), had more satisfying relationships with others, had a positive attitude toward themselves, and were able to manage their lives well and continuously [21]. This was consistent with a research finding that people who had a feeling of closeness to God tended to have global forgiveness, and global forgiveness was a factor associated with the well-being of older adults.

\section{Setting life goals}

Autonomous goals promote well-being. Goal progress is an ideal way to implement this definition of adulthood, as progress in personal goals demonstrates success and autonomous engagement with the physical or social environment. Daily progress on independent goals can increase mental, physical and 
social well-being on the same day and the next day. One report stated that, when older adults made more progress than an average goal of a single day, the following day (as well as concurrently), they would experience a positive impact on sleep quality, relationship quality, and reduction of physical symptoms. A purpose in life is, therefore, correlated with secure attachment and eudemonic well-being by having selfcompassion mediating each of these relationships [22].

\section{Resources supporting older adults}

A literature review of 7 articles disclosed that the main resources supporting well-being and success in older adult lives were classified into 3 sub-groups, namely, family support, social support and religious support.

\section{Family support}

Studies have shown that older adults who have children receive support from their families and live in their own homes, unlike those who have no children. Furthermore, women have reported that they had to get love and friendship from their husbands [12]. This is consistent with the study of Rinnan et al. [14], which suggested that positive relationships and receiving a feeling of being part of a family make the lives of older adults meaningful.

\section{Social support}

Having good friends is the key to a happy life. The older adults in this study emphasized that their social involvement (sports activities) gave them many good friends. It was also found that friends in every age group, local community members, and neighbors were important to social operations, as well as being an important source of daily help for older adults [12]. These reflect that social desire affects the satisfaction of life in older adults and leads to well-being in various aspects of life [23]. Ferguson and Goodwin [20] found that social support appears to mediate the relationship between optimism and subjective well-being in older adults. Meaningful social roles are important in the good lives of older adults, with activities such as caring for children or partners, helping others, being members of an organization, and interacting with society and relationships with others. The abilities to maintain a meaningful social life and perform social activities previously performed in the older adults were also important. The older adults stated that recreation and social entertainment consisted of activities such as hanging out with others, hobbies, listening to music, and visiting family members [13].

\section{Religious support}

There are reasons to think that God is a very important person for older adults. First, the fact that religious beliefs increase according to age is well known. Second, old age eventually brings with it the loss of loved ones, including important figures and former attachments. Many older people do not have parents and have become widowed. These things cause God to become more valuable as a symbol during this age. It has been reported that older adults who have experienced the death of a spouse have increased perceptions of relationships, love, and protection from God [21]. God and church are spiritual supporters in older adults, thereby making sense of security in life. Capable of seeing clearly in times of crisis, many people look up to God as a source of support and consolation, with the belief that they are in God's presence as a safe base. Religion is a center for psychological attachment, especially concerning death. For example, one of the older adults in this study commented, "Thank God for every day that I wake up...because when it's my time, I want to go without knowing I'm gone!" [12]. Religion greatly influences the beliefs of older adults, as reflected by an interviewee who said, "Religion has been a big influence on my change over time in my mode of thinking." [17]. A study by Russo-Netzer and Littman-Ovadia [16] found that being a part of a religious community was a factor that helped eliminate late age-related concerns. The results of another research study showed that time perspective (TP) was related to the well-being of older adults. Furthermore, TP was conceived as a fundamental process, influenced by factors such as culture, religion, social class, education, family modeling and age [24].

\section{Discussion}

In recent years, interest in older wellness has increased, based on different theoretical constructs such as positive aging, healthy aging, active aging and successful aging [25,26]. Although there is some evidence to indicate greater significance among older adults and some enhanced knowledge of factors leading to wellness in older adults, studies from the older perspective on these matters are rare.

This study aimed to explore the experiences of well-being and happiness of older people by using an integrative review. The study will give both qualitative and quantitative research results that are related 
to the well-being and happiness of older adults. The qualitative research results provide direct perspectives and experiences from the insights of specific people, while the quantitative research results confirm the relevance and relationships of various factors that affect the well-being and happiness of older adults. Therefore, the educational results are comprehensive, holistic and clear in all dimensions.

The results revealed the following 3 issues: (a) the meaning of well-being in older adults in relation to physical, psychological, emotional, social and spiritual dimensions; (b) the meaning of well-being and happiness in older adults- from the reviews relevant to the meaning of well-being and happiness in older adults, happiness is primarily characterized as a state of well-being that contributes to wellness in the older population. Well-being and physical and mental health are closely related, and this relationship may become more significant in older adults, contributing to older adults' well-being in terms of life satisfaction, feelings of happiness, and maintaining a sense of purpose and value in life [27,28]; (c) the key viewpoints of 3 main categories (challenges and concerns of life, strategies for a meaningful life, resources and support), and 13 sub-categories on well-being and happiness in older adults, which provided interesting viewpoints regarding the following information.

The $1^{\text {st }}$ viewpoint that could be outlined from several literature reviews about the well-being of older adults with the story that the effects of life at this age differing from past life stages. In particular, stories about death were the biggest concern of older adults, who have a universal need to meet a good death. Gerstorf et al. [29] suggested that mortality-related mechanisms drove late-life changes in wellbeing, finding that approaching death in older adults led to a decrease in well-being. The $2^{\text {nd }}$ viewpoint was concerned with a variety of strategies that helped older adults be happy and well. It was found that performing a daily routine, self-esteem, life satisfaction, engagement and subjectively perceived activities, optimism, attachment to God, and goals in life could help increase self-worth in older adults. Optimism might influence mental and physical well-being by promoting a healthy lifestyle [30]. Herero and Extremera [31] stated, "Mediational analyses revealed that social activities partially mediated the relationship between personality variables - self-esteem and optimism - and subjective well-being." The last viewpoint was about the 3 main sources of support that were important to older adults' well-being, which were family support, social support and religious support. Nguyen et al. [32] found that social support (family and friends) made unique contributions to the well-being of older African Americans. It was discovered by the research that adolescent and/or adult grandchildren's social support played a major role in contributing to life satisfaction for older adults in Hong Kong [33]. Park et al. [34] found that "greater religiosity was related to greater life satisfaction and social support partially explained the positive relationship between religiosity and life satisfaction." The results of Lee's study are similar in that forgiveness and religious support were found to play key roles in older adults' well-being [35]. Therefore, it is also important to keep in mind older adults' social and cultural backgrounds when considering their life well-being and happiness. Differences in race, ethnicity, traditions, beliefs, values, geographical features, and economic status definitely affect definitions of well-being and happiness in older adults from different areas.

According to Maslow's Hierarchy of Needs philosophy, older persons focus on current needs in life, which vary from past life experience to the present day [36]. The findings of this study revealed a 5-step model for well-being and happiness in older adults, addressing biological and physiological needs, safety needs, social needs, esteem needs and self-actualization needs [37]. Moreover, the above literature review suggested that older adults need nutrition, hygiene, exercise or movement and a warm, sheltered place. The safety needs of older adults mean protection from social abuse and receipt of necessary health care and health insurance, whether the older adults are independent and relatively healthy or suffering from illness. Social needs for older adults involve relationships with family and friends, social interactions outside the family, and contact with other people [16]. Maintaining self-esteem and being respected is also very important for older adults' happiness and well-being, because they might also feel less valued when their independence and skills diminish. It was found that most of the needs of this age focused on finding a meaning in life or needs of self-actualization, which means self-awareness of personal potential, selffulfillment, pursuit of personal growth, and peak experience. The findings of Wang [38] showed that the needs of older adults varied according to individual identity, as reflected in the quote, "The basic needs are for survival, self-fulfillment, family needs, communications, personalized activities and encouragement, all of which might have a conductive function for older people to live a happy later life." 


\section{Conclusions}

Based on situations around the world, the older population is increasing steadily. The question of how to create independence and freedom in the lives of older adults is a very challenging and interesting issue for the next century. According to the above, it can be seen that experiences and perceptions of health among older adults describe how to create their well-being and happiness, which truly depends on the original experiences and the basis of individual lives. However, this knowledge from the experiences of older adults will enable professional nurses to target health and well-being among individual older adults more precisely. Further research is needed to understand how to facilitate and enhance well-being and happiness in older adult lives. Nevertheless, professional nurses can use this study as basic information in designing guidelines, developing new care models, and giving interventions to increase the efficiency of care in older adult populations.

\section{Acknowledgements}

The researchers would like to thank the Faculty of Nursing, Thammasat University, Thailand, for the opportunity to learn and develop this academic nursing article.

\section{References}

[1] Institute for Population and Social Research, Mahidol University. Situation of the Thai elderly, Available at: https://op.mahidol.ac.th/ra/2017/11/25/pr-01/, accessed March 2020.

[2] S McMahon and J Fleury. Wellness in older adults: A concept analysis. Nurs. Forum 2012; 47, 3951.

[3] M Cattan. Mental health and well-being in later life. McGraw-Hill Education, New York, 2009.

[4] AL Collins, N Sarkisian and E Winner. Flow and happiness in later life: An investigation into the role of daily and weekly flow experiences. J. Happiness Stud. 2009; 10, 703-19.

[5] V Gonzalez-Herero and MA Garcia-Martin. Personality, activities, and well-being: A study based on women in late adulthood. J. Women Aging 2012; 24, 156-68.

[6] MTD Souza, MDD Silva and RD Carvalho. Integrative review: What is it? How to do it? Einstein (Sao Paulo). 2010; 8, 102-6.

[7] R Whittemore and K Knafl. The integrative review: updated methodology. J. Adv. Nurs. 2005; 52, 546-53.

[8] The Joanna Briggs Institute. Reviewers' manual: 2014 edition The Joanna Briggs Institute, Australia, 2014.

[9] The Joanna Briggs Institute. The Joanna Briggs Institute EBP database guide, Available at: https://joannabriggs.org/ebp/critical appraisal tools, accessed May 2020.

[10] A Liberati, DG Altman, J Tetzlaff, C Mulrow, $\bar{P}$ C Gotzsche, JPA Ioannidis, M Clarke, PJ Devereaux, J Kleijnen and D Moher. The PRISMA statement for reporting systematic reviews and meta-analyses of studies that evaluate healthcare interventions: Explanation and elaboration. BMJ 2009; 339, b2700.

[11] MJ Page, JE McKenzie, PM Bossuyt, I Boutron, TC Hoffmann, CD Mulrow, L Shamseer, JM Tetzlaff, EA Akl, SE Brennan, R Chou, J Glanville, JM Grimshaw, A Hróbjartsson, MM Lalu, T Li, EW Loder, E Mayo-Wilson, S McDonald, LA McGuinness, LA Stewart, J Thomas, AC Tricco, VA Welch, P Whiting, D Moher. The PRISMA 2020 statement: An updated guideline for reporting systematic reviews. BMJ 2021; 372, n71.

[12] N Hutnik, P Smith and T Koch. What does it feel like to be 100? Socio-emotional aspects of wellbeing in the stories of 16 centenarians living in the United Kingdom. Aging Ment. Health 2012; 16, $811-8$.

[13] L Nosraty, M Jylha, T Raittila and K Lumme-Sandt. Perceptions by the oldest old of successful aging, vitality 90+ study. J. Aging Stud. 2015; 32, 50-8.

[14] E Rinnan, B Andre, J Drageset, H Garasen, GA Espnes and G Haugan. Joy of life in nursing homes: a qualitative study of what constitutes the essence of joy of life in elderly individuals living in Norwegian nursing homes. Scand. J. Caring Sci. 2018; 32, 1468-76.

[15] T Kavcic and A Avsec. A day in the lives of older adults: What makes them happy? Psiholoska Obzorja/Horizons Psychol. 2018; 27, 51-60.

[16] P Russo-Netzer and H Littman-Ovadia. "Something to live for": Experiences, resources and personal strengths in late adulthood. Front. Psychol. 2019; 10, 2542.

[17] AI Hoogland. Continuity of change: The dynamic of beliefs, values, and the aging experience. $J$. Aging Stud. 2015; 32, 32-9. 
[18] P Juneja. Strategy - definition and features, Available at: https://www.managementstudyguide.com/ strategy-definition.htm, accessed April 2020.

[19] XAS Roman, MC Toffoletto, JCO Sepulveda, SV Salfate and KLR Grandon. Factors associated with subjective well-being in older adults. Texto Contexto Enferm. 2017; 26, e5460015.

[20] SJ Ferguson and AD Goodwin. Optimism and well-being in older adults: The mediating role of social support and perceived control. Int. J. Aging Human Dev. 2010; 71, 43-68.

[21] KJ Homan. Symbolic attachment security and eudemonic well-being in older adults. J. Adult Dev. $2014 ; 21,89-95$.

[22] KJ Homan. Secure attachment and eudaimonic well-being in late adulthood: The mediating role of self-compassion. Aging Ment. Health. 2018; 22, 363-70.

[23] MC Fastame, MP Penna and PK Hitchcott. Life satisfaction and social desirability across the late life span: What relationship? Qual. Life Res. 2015; 24, 241-4.

[24] F Desmyter, and RD Raedt. The relationship between time perspective and subjective well-being of older adults. Psychol. Belg. 2012; 52, 19-38.

[25] C Guell, J Panter, S Griffin and D Ogilvie. Towards co-designing active ageing strategies: a qualitative study to develop a meaningful physical activity typology for later life. Health Expect. 2018; 21, 919-26.

[26] S Eloranta, S Arve, H Isoaho, A Lehtonen and M Viitanen. Factors connected with positive life orientation at age 70, 80, 85 and 90 - the Turku elderly study. Scand. J. Caring Sci. 2015; 29, 53747.

[27] CD Ryff. Psychological well-being in adult life. Curr. Dir. Psychol. Sci. 1995; 4, 99-104.

[28] A Steptoe, A Deaton and AA Stone. Subjective well-being, health and ageing. Lancet 2015; 385, 640-8.

[29] D Gerstorf, N Ram, G Mayraz, M Hidajat, U Lindenberger, GG Wagner and J Schupp. Late-life decline in well-being across adulthood in Germany, the United Kingdom, and the United States: Something is seriously wrong at the end of life. Psychol. Aging 2010; 25, 477-85.

[30] C Conversano, A Rotondo, E Lensi, O Della Vista, F Arpone and MA Reda. Optimism and its impact on mental and physical well-being. Clin. Pract. Epidemiol. Ment. Health 2010; 6, 25-9.

[31] VG Herero and N Extremera. Daily life activities as mediators of the relationship between personality variables and subjective well-being among older adults. Pers. Indiv. Differ. 2010; 49, 124-9.

[32] AW Nguyen, LM Chatters, RJ Taylor and DM Mouzon. Social support from family and friends and subjective well-being of older African Americans. J. Happiness Stud. 2016; 17, 959-79.

[33] VW Lou. Life satisfaction of older adults in Hong Kong: The role of social support from grandchildren. Soc. Indicat. Res. 2010; 95, 377-91.

[34] J Park, S Roh, and Y Yeo. Religiosity, social support, and life satisfaction among elderly Korean immigrants. Gerontologist 2012; 52, 641-9.

[35] KH Lee. The role of spiritual experience, forgiveness, and religious support on the general wellbeing of older adults. J. Relig. Spirituality Aging 2011; 23, 206-23.

[36] AH Maslow. Motivation and Personality. Harper \& Row, New York, 1970.

[37] A McLeod. Maslow's Hierarchy of Needs, Available at: http://www.simplypsychology.org/ maslow.html, accessed March 2020.

[38] Q Wang. The needs of older people in later life, Available at: https://www.divaportal.org/smash/get/diva2:575674/FULLTEXT01.pdf, accessed March 2020. 